\title{
Bringing a Fourth Order Autonomous Inverter Power Network into Such of a Second Order in a Normalized Form
}

\author{
Evgeniy I. Popov ${ }^{1}$, Nikolay L. Hinov ${ }^{1}$ \\ ${ }^{I}$ Department of Power Electronics, Technical University of Sofia, \\ Bul Kl. Ohridski 8,1000 Sofia, Bulgaria \\ epopov@tu-sofia.bg
}

\begin{abstract}
A method is developed for reducing the power inverter electrical circuit of fourth order to an electrical network of second order. The reduction is based on the assumption that the output inverter voltage is practically with a sine-wave shape. The normalized form guarantees novelty and versatility of the investigation on one hand and independency in relation to the concrete inverter parameters like output power, supplying voltage and operating frequency on the other. From the whole power network of fourth order for the first time two sub circuits are suggested to be outlined: the output (load) sub circuit and the inverter commutating sub circuit. Both sub circuits of a second order define the parameters of the endmost inverter circuit again of the same order and consequently the mode of operation and parameters of the power inverter itself. The transformation is possible owing to the newly found fact that the frequency dependencies of the output (load) sub circuit can be separated from the dependencies that describe the structural relationships between the two sub circuits. The results from that conversion are of course more accurate when the resonant frequencies of the both sub circuits and the operating frequency do not differ substantially.

The suggested approach is useful on order to obtain a simplified engineer method for precise and fast design of large class of static power converters applicable in electronic technology like induction heating and melting.
\end{abstract}

Index Terms-Autonomous inverter, fourth order, normalised form, power circuit, second order.

\section{INTRODUCTION}

From many years of research in the area of computer aided analysis and design of power electronic circuits and from the practice of application of autonomous inverters for electro technology, for instance in the area of induction heating, follows that very often a fundamental component of the inverter power circuits is an electrical network of fourth order [1]-[3]. It is difficult to achieve a direct analytical solution of such a high order electrical circuit and in most cases that process is avoided. That is why the inverter electrical circuit of fourth order is reduced to an inverter electrical network of a second order that principally has an analytical solution for the similar processes taking place inside the power inverter [4], [5]. From that a whole set of methods [6], [7] follows for quick determination of the modes, parameters and the whole necessary information describing the corresponding power autonomous serial RLC inverter with or without free - wheeling diodes. The reduction of the inverter network is based on the assumption close to the real case that the output voltage of the inverter appearing across the load inductor and the connected in parallel output capacitor (namely the output parallel resonant circuit) is practically with a sine-wave shape due to the relatively high quality factor of the output inverter circuit.

It is important that bringing of the fourth order inverter network into a such of a second order to be implemented in a normalized form in order to achieve in a newly suggested way versatility of the investigation and independency in relation to the concrete inverter parameters like output power, supplying voltage and operating frequency, which is the aim of the present article.

The principal inverter fourth order electrical power circuit is shown in Fig. 1. $L_{k}$ and $C_{k}$ are the commutating inductance and capacitance. $L_{l}$ and $R_{l}$ are the inductance and resistance of the parallel equivalent circuit of the load inductor itself.

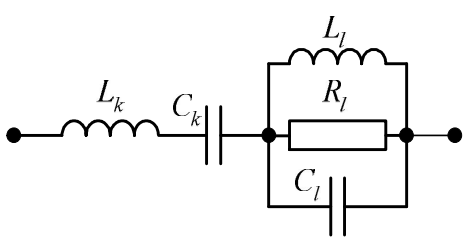

Fig. 1. The fourth order electrical power circuit.

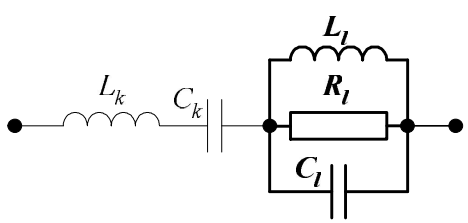

Fig. 2. The parallel output (load) sub circuit.

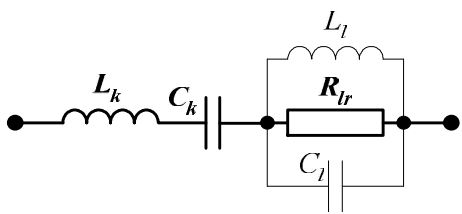

Fig. 3. The inverter commutating serial sub circuit.

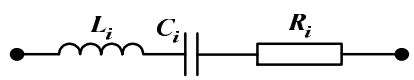

Fig. 4. The second order endmost inverter circuit. 
It is proposed that from the whole power network (of 4th order) two sub circuits to be outlined - parallel (for instance) output (load) sub circuit (Fig. 2) and the inverter serial (for instance) sub circuit (Fig. 3). $C_{l}$ is the capacitance of the output (load) capacitor. Both sub circuits (Fig. 2 and Fig. 3) define the parameters of the endmost inverter circuit of 2 nd order (Fig. 4) and consequently the mode of operation and parameters of the power inverter itself. It should be said in advance that the results from that conversion will be much more accurate when the resonant frequencies of the both sub circuits (Fig. 2 and Fig. 3) do not differ substantially taking into account the influence of their also different quality factors. The inverter operating frequency should be close to the already mentioned resonant frequencies. But further investigations are necessary to be carried out in order to be able to say something much more definitely about the accuracy in a given instance. This transformation is possible owing to the fact that the frequency dependencies of the output (load) sub circuit can be separated from the dependencies that describe the structural relationships between the two sub circuits [8], [9].

\section{THE FREQUENCY DEPENDENCIES OF THE PARALLEL OUTPUT (LOAD) SUB CIRCUIT}

If the inductor is represented by a parallel equivalent circuit and taking into account the almost sine wave shape of the voltage across it, the frequency dependency of its parameters resistance $R_{1}$ and inductance $L_{l}$ are:

$$
\begin{gathered}
R_{l}=R_{l r}\left(\omega / \omega_{l r}\right)^{n_{1}}, \\
L_{l}=L_{l r}\left(\omega / \omega_{l r}\right)^{n_{2}-1},
\end{gathered}
$$

where $\omega$ is the controlling angular frequency and $\omega_{\mathrm{lr}}$ is the resonant angular frequency of the output (load) sub circuit $\mathrm{R}_{\mathrm{lr}}, \mathrm{L}_{\mathrm{lr}}, \mathrm{C}_{\mathrm{l}}$ (Fig. 2). $\mathrm{R}_{\mathrm{lr}}, \mathrm{L}_{\mathrm{lr}}$ are the parameters for the resonant frequency (index $r$ ). $\mathrm{n}_{1}, \mathrm{n}_{2}$ are defined from the geometry of the system inductor - heated piece, and from the physical parameters of the heated piece itself. Their values for non magnetic and ferromagnetic are given in [1]. The quality factor of the output (load) sub circuit $\mathrm{R}_{\mathrm{l}}, \mathrm{L}_{\mathrm{l}}, \mathrm{C}_{\mathrm{l}}$ at resonance is

$$
Q_{l r}=R_{l r} \sqrt{C_{l} / L_{l r}}
$$

Then the parameters of the serial equivalent circuit of the output (load) sub circuit are (the transformer ratio is also taken into account):

$$
\begin{gathered}
\frac{R_{e q}}{R_{l r}}= \\
=\frac{\left(\omega / \omega_{l r}\right)^{n_{1}}}{1+Q_{l r}^{2}\left(\left(\omega / \omega_{l r}\right)\right)^{\left(2 n_{1}-n_{2}+1\right)}\left(\left(\omega / \omega_{l r}\right)-1 /\left(\omega / \omega_{l r}\right)\right)^{2}}= \\
=F_{R}\left(\omega / \omega_{l r}, Q_{l r}\right), \\
\frac{X_{e q}}{R_{l r}}= \\
=\frac{Q_{l r}\left(\omega / \omega_{l r}\right)^{\left(2 n_{1}+\frac{1-n_{2}}{2}\right)}\left(1 /\left(\omega / \omega_{l r}\right)-\left(\omega / \omega_{l r}\right)\right)}{1+Q_{l r}^{2}\left(\omega / \omega_{l r}\right)^{\left(2 n_{1}-n_{2}+1\right)}\left(\left(\omega / \omega_{l r}\right)-1 /\left(\omega / \omega_{l r}\right)\right)^{2}}=
\end{gathered}
$$

$$
=F_{X}\left(\omega / \omega_{l r}, Q_{l r}\right)
$$

$\omega / \omega_{l r}, \quad Q_{l r}$ could be determined if the following fundamental parameters $(\mu, \lambda)$ or $(\cos \varphi, B)$ of the output (load) sub circuit are known in advance:

$$
\begin{gathered}
\omega / \omega_{l r}=\mu_{O}=\left(\sqrt{B_{O} \sqrt{1-\cos ^{2} \varphi_{O}}}\right)^{-1}, \\
Q_{l r}=1 /\left(2 \lambda_{O}\right)=\sqrt[4]{1-\cos ^{2} \varphi_{O}} /\left(\sqrt{B_{O}} \cos \varphi_{O}\right), \\
\cos \varphi_{O}=\frac{\omega L_{l r}}{\sqrt{R_{l r}^{2}+\omega^{2} L_{l r}^{2}}}, \\
B_{O}=\frac{\sqrt{R_{l r}^{2}+\omega^{2} L_{l r}^{2}}}{\omega^{2} L_{l r} C_{l} R_{l r}}=\frac{1}{\omega C_{l} R_{l r} \cos \varphi_{O}}, \\
\mu_{O}=\omega \sqrt{L_{l r} C_{l}}=\omega / \omega_{l r}, \\
\lambda_{O}=\frac{1}{2 R_{l r}} \sqrt{\frac{L_{l r}}{C_{l}}}=1 /\left(2 Q_{l r}\right) .
\end{gathered}
$$

The frequency dependent coefficients can be calculated:

$$
\begin{aligned}
& K_{O}=\left\{\begin{array}{l}
0 \Rightarrow \omega / \omega_{l r} \leq 1 \\
1 \Rightarrow \omega / \omega_{l r}>1
\end{array}\right\}, \\
& K_{O 1}=\frac{1}{\left(\omega / \omega_{l r}\right)} \times \frac{1}{F_{R}\left(\omega / \omega_{l r}, Q_{l r}\right)}, \\
& K_{O 2}=K_{O} \frac{F_{X}\left(\omega / \omega_{l r}, Q_{l r}\right)}{F_{R}\left(\omega / \omega_{l r}, Q_{l r}\right)}, \\
& K_{O 3}=\frac{\omega / \omega_{l r}}{F_{R}\left(\omega / \omega_{l r}, Q_{l r}\right)}, \\
& K_{O 4}=\left(1-K_{O}\right) \frac{F_{X}\left(\omega / \omega_{l r}, Q_{l r}\right)}{F_{R}\left(\omega / \omega_{l r}, Q_{l r}\right)}, \\
& K_{O 5}=\left\{\begin{array}{l}
\left(\omega / \omega_{l r}\right) \times \frac{1}{F_{X}\left(\omega / \omega_{l r}, Q_{l r}\right)} \Rightarrow \omega / \omega_{l r} \leq 1 \\
-\left(\omega_{l r} / \omega\right) \times \frac{1}{F_{X}\left(\omega / \omega_{l r}, Q_{l r}\right)} \Rightarrow \omega / \omega_{l r}>1
\end{array}\right\}, \\
& K_{O 2,4}=\left\{\begin{array}{ll}
K_{O 4} & \Rightarrow \omega / \omega_{l r} \leq 1 \\
-K_{O 2} & \Rightarrow \omega / \omega_{l r}>1
\end{array}\right\} .
\end{aligned}
$$

\section{THE PARAMETERS OF THE INVERTER COMMUTATING SERIAL SUB CIRCUIT}

The power factor and the load coefficient of the inverter serial sub circuit $\mathrm{R}_{\mathrm{k}}, \mathrm{L}_{\mathrm{k}}, \mathrm{C}_{\mathrm{k}}$ (Fig. 3) are:

$$
\begin{gathered}
\cos \varphi_{K}=\frac{\omega C_{k} R_{l r}}{\sqrt{1+\omega^{2} C_{k}^{2} R_{l r}^{2}}} \\
B_{K}=\frac{\sqrt{1+\omega^{2} C_{k}^{2} R_{l r}^{2}}}{\omega^{2} L_{k} C_{k}}=\frac{R_{l r}}{\omega L_{k} \cos \varphi_{K}} .
\end{gathered}
$$

The frequency coefficient is

$$
n_{K}=\frac{2}{\sqrt{4 B_{K} \sqrt{1-\cos ^{2} \varphi_{K}}-B_{K}^{2} \cos ^{2} \varphi_{K}}}
$$


The coefficient of hesitation is

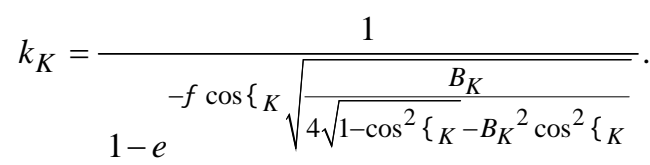

\section{The PARAmEters OF THE SECOND ORdER ENDMOST INVERTER CIRCUIT}

The parameters of the second order endmost inverter circuit $L_{i}, C_{i}, R_{i}$ (Fig. 4), based on the structural relationships between the two circuits (output - load on one hand and the commutating - inverter on the other) are $\tan \varphi_{I}=K_{O 1} \tan \varphi_{K}+K_{O} K_{O 2,4}=K_{O 1} \tan \varphi_{K}-K_{O 2}$, where (24):

$$
\begin{gathered}
\tan \varphi_{K}=\sqrt{1-\cos ^{2} \varphi_{K}} / \cos \varphi_{K}, \\
\cos \varphi_{I}=1 / \sqrt{1+\tan ^{2} \varphi_{I}}, \\
B_{I}=\frac{1}{\frac{K_{O 3}}{B_{K} \cos \varphi_{K}}+\left(1-K_{O}\right) K_{O 2,4}} \times \frac{1}{\cos \varphi_{I}} .
\end{gathered}
$$

Then, according to [6], [7] and [10] for oscillatory mode of the inverter circuit $\left(R_{i}<2 \sqrt{L_{i} / C_{i}}\right)$ the coefficient is $\mathrm{c}=$ 1 , for over damped mode $\left(R_{i}>2 \sqrt{L_{i} / C_{i}}\right) c=-1$ and for critical mode $\left(R_{i}=2 \sqrt{L_{i} / C_{i}}\right) c=0$. The parameters that determine the development of the electromagnetic processes in steady state mode in the power inverter are

$$
\begin{gathered}
n_{\delta I}=n_{\delta}=\frac{\delta}{\Omega}=\frac{B_{I} \cos \varphi_{I}}{\sqrt{c\left(4 B_{I} \sin \varphi_{I}-B_{I}^{2} \cos ^{2} \varphi_{I}\right)}}, \\
\text { (osc.; over damped) } ; 1 \text { (critical) },
\end{gathered}
$$

For resonant inverters (oscillatory mode) the coefficient of hesitation is

$$
k_{I}=1 /\left[1-\exp \left(-\pi \delta / \omega_{0}\right)\right]=1 /[1-\exp (-\pi \delta / \Omega)]
$$

The frequency coefficient is

$$
\begin{gathered}
n_{\omega I}=n_{\omega}=\frac{\omega}{\Omega}=\frac{2}{\sqrt{c\left(4 B_{I} \sin \varphi_{I}-B_{I}^{2} \cos ^{2} \varphi_{I}\right)}}, \\
\text { (osc.; over damped }) ; \frac{2}{B_{I} \cos \varphi_{I}}(\text { critical }) .
\end{gathered}
$$

The following additional parameters can be determined

$$
\begin{array}{r}
\beta_{I}=\frac{1}{1+\frac{K_{O 5}}{B_{K} \cos \varphi_{K}}}, \\
b_{I}=\frac{1+\frac{b K_{O 5}}{B_{K} \cos \varphi_{K}}}{1+\frac{K_{O 5}}{B_{K} \cos \varphi_{K}}},
\end{array}
$$

$$
\begin{gathered}
\alpha_{I}=0 \Rightarrow \omega / \omega_{l r} \leq 1, \\
\beta_{I}=0, \\
b_{I}=b, \\
\alpha_{I}=\frac{1}{1+K_{O 5} \tan \varphi_{K}} \Rightarrow \omega / \omega_{l r}>1,
\end{gathered}
$$

where $\beta_{I} / \alpha_{I}$ are the coefficients of inductive/capacitive detuning of the output (load) sub circuit, $b_{I}$ is the coefficient of a real distribution of the commutating inductance between the diagonal and the input circuit in a bridge configuration of the inverter, $b$ is the same at resonance.

\section{AN EXAMPLE WITH A SERIAL RLC RESONANT INVERTER WiTHOUT FREE WHEELING DiODES}

The mode of operation parameters of the mentioned type of inverter are studied on the basis of the transformation already suggested. The inverter initial data correspond to ( $b=0.5 ; n_{K}=0,8508 ; k_{K}=1,5185$ - a practically verified case from another article by the authors).

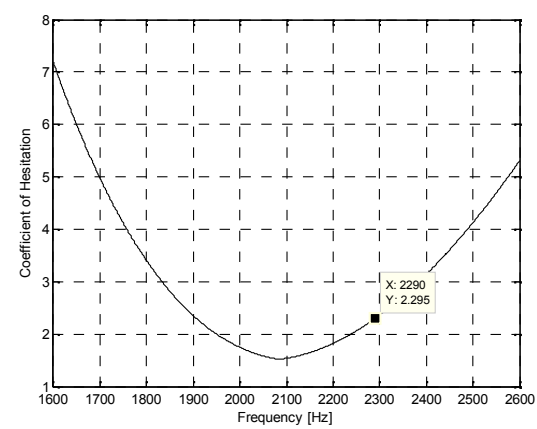

Fig. 5. 2D graphic: Coefficient of hesitation as a function of frequency.

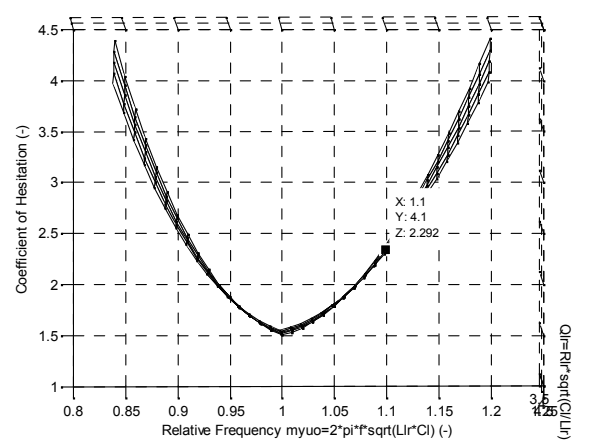

Fig. 6. 3D graphic: Coefficient of hesitation as a function of the relative frequency and the quality factor at resonance of the output (load) sub circuit.

The result for $k_{I}$ from a MATLAB inverter analysis and a 2D graphic for the example under consideration (it has a PSPICE confirmation as well) is compared with the 3D MATLAB graphic obtained from the proposed method (Fig. 5 and Fig. 6). The observed error is not larger than $3 \%$.

The parameters of the output (load) sub circuit are varied $\omega / \omega_{l r}=0,84 \div 1,2 ; Q_{l r}=2 \div 8$. The following inverter parameters are observed $\left(n_{I} ; k_{I} ; \alpha_{I} ; \beta_{I} ; b_{I}=f\left(\omega / \omega_{l r}, Q_{l r}\right)\right.$. From them it can be determined: 1) Concrete inverter parameters at the mode of 
operation; 2) Influence of the output (load) sub circuit parameter variations on the whole inverter operation.

\section{AN EXAMPLE WITH A SERIAL RLC RESONANT INVERTER WITH FREE WHEELING DIODES}

The result for $\alpha_{I}$ from an example ( $b=0 ; n_{K}=0,89484 ; k_{K}=1,9026$ - a practically verified case from another article by the authors), $\left(\omega / \omega_{l r}=0,84 \div 1,2 ; Q_{l r}=2 \div 8\right), \quad$ a MATLAB inverter analysis together a $2 \mathrm{D}$ graphic is compared with the $3 \mathrm{D}$ MATLAB graphic obtained from the proposed method transformation (Fig. 7 and Fig. 8).

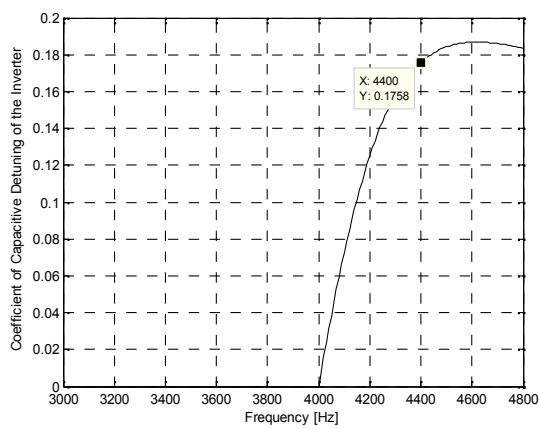

Fig. 7. 2D graphic: Coefficient of capacitive detuning of the inverter as a function of frequency.

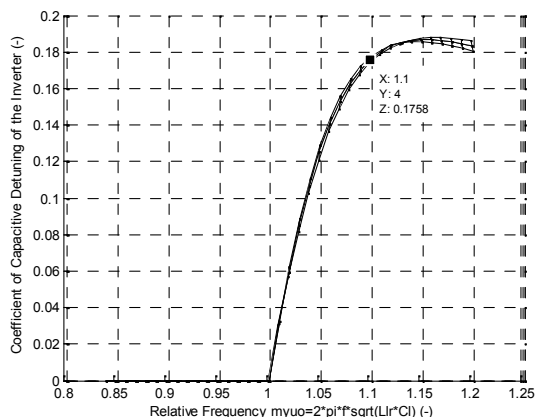

Fig. 8. 3D graphic: Coefficient of capacitive detuning of the inverter as a function of the relative frequency and the quality factor at resonance.

Tested circuit with reverse conducing diodes is shown in Fig. 9.

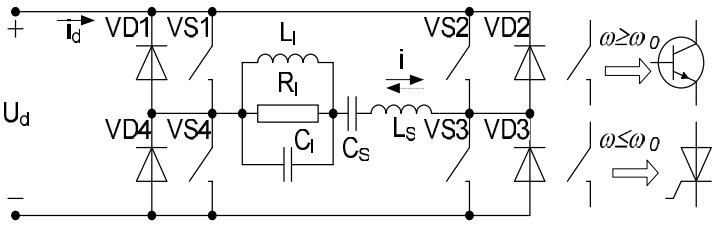

Fig. 9. Tested circuit with reverse conducing diodes.

Parameters are $\mathrm{U}_{\mathrm{d}}=500[\mathrm{~V}] ; \mathrm{L}_{\mathrm{S}}=0.3[\mathrm{mH}] ; \mathrm{C}_{\mathrm{S}}=4[\mu \mathrm{F}]$; $\mathrm{C}_{1}=39.789[\mu \mathrm{F}]$; Load: induction heater or melter, uncertain parameters, assumed in the case: $\mathrm{R}_{1}=4\left[\mathrm{]} ; \mathrm{L}_{\mathrm{l}}=\right.$ $39.789[\mu \mathrm{H}]$; Frequencies: operating (controllable): $\mathrm{f}=$ $4000[\mathrm{~Hz}] \pm 30 \%$; inherent of the commutating inverter serial sub circuit (changeable, calculated in the case): $\mathrm{f}_{0}=$ $4470[\mathrm{~Hz}]$.

Many comparisons were made for $n_{u} ; k_{u} ; \alpha_{u} ; \beta_{u} ; b_{u}$ (inverters type V and VI each supported by a MATLAB investigation, PSPICE confirmation and experimentally) on one hand and the suggested method on the other. Again the observed error is not larger then $3 \%$.

\section{CONCLUSIONS}

A new method is proposed for reduction of a fourth order electrical power inverter network to such of a second order for brief, easy determination of the set of parameters describing the operation of the practical inverter. The method is developed in a normalized form making it versatile to the concrete inverter parameters. The results are more accurate if the degree of detuning of the inverter circuits is not large but further investigations should clarify the issue.

Such a transformation is possible because the frequency dependencies of the output (load) sub circuit can be separated from the dependencies that describe the structural relationships between the two sub circuits (output/load and commutating/serial).

The method is applied for complete examination of serial resonant inverters without or with freewheeling diodes not dependent of the concrete inverter parameters. It is also applicable for examination of parallel and serial parallel current fed inverters, and a modification in case of a parallel serial current fed inverter with an increase of the output voltage. The perceived error is not larger than $3 \%$. The presented approach is useful in the implementation of the controlling system of power converters operating in switch mode [11], [12]. This aspect is very important for induction technologies where the loads have large parameter variations during the real technology process [13].

\section{REFERENCES}

[1] E. I. Berkovitch, G. V. Ivenskiy, Yu. S. Yoffe, A. T. Matchak, V. V. Morgun, "Higher frequency thyristor converters for electro technological units". St. Petersborough: Energoatomizdat, 1983. (in Russian).

[2] G. I. Atabekov, "Fundamentals of circuit theory". Moscow: Energy, 1969. (in Russian)

[3] N. Al. Nachev, G. Yu. Maleev, "Power Electronics". Sofia: Technica, 1979. (in Bulgarian)

[4] N. L. Hinov, "Power converters of electrical energy with industrial application", Ph.D. Dissertation, Technical University, Sofia, 1998 , (In Bulgarian).

[5] N. Balabanian, T. A. Bickart, "Electrical network theory". New York: John Willey, 1969

[6] E. I. Popov, "Investgation and Analysis of Serial Thyristor RLC Inverters", Electrical Engineering and Electronics E+E, vol. 7-9, pp. 39-43, 2003. (in Bulgarian).

[7] E. I. Popov, N. P. Kolev, "Investigation and analysis of semiconductor RLC inverters", Technical ideas, vol. 39, no. 1-2, pp. 34-43, 2002.

[8] C. W. Lander, "Power Electronics". Mc Graw - Hill Book Company, 1993.

[9] N. Mohan, T. M. Undeland, W. P. Robbins, "Power electronics, converters, applications and design”. John Wiley \& Sons, 2003.

[10] E. I. Popov, "Direct determination of the steady-state mode in autonomous inverters", Electrical Engineering and Electronics $E+E$, vol. 11-12, 2004. (in Bulgarian).

[11] D Pikulins, "Exploring types of instabilities in switching power converters: the complete bifurcation analysis", Elektronika ir Elektrotechnika, vol. 20, no. 5, pp. 76-78, 2014. [Online]. Available: http://dx.doi.org/10.5755/j01.eee.20.5.7103

[12] D. Stepins, J. Jankovskis, "Study of Frequency Modulated Boost Converter Operating in Discontinuous Conduction Mode", Elektronika ir Elektrotechnika, no. 6, pp. 41-44, 2012. [Online]. Available: http://dx.doi.org/10.5755/j01.eee.122.6.1819

[13] E. I. Popov, "Automatic Control of Serial Thyristor Inverters with Varying Load”, Ph.D. Thesis, VMEI, Sofia, 1978. (in Bulg rian). 